\title{
Structural and electronic properties of solid molecular hydrogen from many-electron theories
}

\author{
Ke Liao $\oplus^{*}$ \\ Max Planck Institute for Solid State Research, Heisenbergstrasse 1, 70569 Stuttgart, Germany \\ Tong Shen $\oplus^{*}$ \\ State Key Laboratory for Artificial Microstructure and Mesoscopic Physics, Frontier Science Center for Nano-optoelectronics \\ and School of Physics, Peking University, Beijing 100871, China \\ Xin-Zheng Li \\ State Key Laboratory for Artificial Microstructure and Mesoscopic Physics, Frontier Science Center for Nano-optoelectronics \\ and School of Physics, Peking University, Beijing 100871, China \\ and Collaborative Innovation Center of Quantum Matter, Peking University, Beijing 100871, China \\ Ali Alavi \\ Max Planck Institute for Solid State Research, Heisenbergstrasse 1, 70569 Stuttgart, Germany \\ and Department of Chemistry, University of Cambridge, Lensfield Road, Cambridge CB2 1EW, United Kingdom \\ Andreas Grüneis $^{\dagger}$ \\ Institute for Theoretical Physics, TU Wien, Wiedner Hauptstraße 8-10/136, 1040 Vienna, Austria
}

(Received 11 September 2020; revised 29 January 2021; accepted 1 February 2021; published 19 February 2021; corrected 2 March 2021)

\begin{abstract}
We study the structural and electronic properties of phase III of solid hydrogen using accurate many-electron theories and compare the results to state-of-the-art experimental findings. The atomic structures of phase III modeled by $C 2 / c-24$ crystals are fully optimized on the level of second-order perturbation theory, demonstrating that previously employed structures optimized on the level of approximate density functionals exhibit errors in the $\mathrm{H}_{2}$ bond lengths that cause significant discrepancies in the computed quasiparticle band gaps and vibrational frequencies compared to experiment. Using these optimized atomic structures, we study the band gap closure and change in vibrational frequencies as a function of pressure. Our findings are in good agreement with recent experimental observations and may prove useful in resolving long-standing discrepancies between experimental estimates of metallization pressures possibly caused by disagreeing pressure calibrations.
\end{abstract}

DOI: 10.1103/PhysRevB.103.054111

\section{INTRODUCTION}

The seminal work of Wigner and Huntington, which first predicted a metallization of hydrogen [1] in 1935 at a pressure of about $25 \mathrm{GPa}$, has sparked continuous interest in the pressure-temperature phase diagram of hydrogen. However, state-of-the-art experiments [2-4] have not been able to conclusively detect metallic behavior with the exception of some recent experimental studies [5-7] that are still under debate $[8,9]$. Until today, one of the most reliable experimental estimates for the metallization pressure range is approximately

\footnotetext{
${ }^{*}$ These authors contributed equally to this work.

${ }^{\dagger}$ Corresponding author: andreas.grueneis@tuwien.ac.at
}

Published by the American Physical Society under the terms of the Creative Commons Attribution 4.0 International license. Further distribution of this work must maintain attribution to the author(s) and the published article's title, journal citation, and DOI. Open access publication funded by the Max Planck Society.
425-450 GPa [7]. The lower value was obtained by the discontinuous pressure evolution in the infrared absorption, assuming a structural phase transition to the atomic structure, whereas the higher value was obtained by extrapolation of the band gap, assuming hydrogen remains in phase III. Determining the metallization pressure accurately is extremely challenging. This is partly reflected by the disagreement of the measured $\mathrm{H}_{2}$ vibron frequency peaks as a function of the pressure, which is crucial for pressure calibration in many experiments [9]. In addition to the electronic structure, questions concerning the atomic structure are also difficult to address. Using x-ray scattering to determine the crystal structure experimentally is hampered by the low scattering cross section of hydrogen. Depending on pressure and temperature, hydrogen has been predicted to condense in different orientationally ordered molecular crystals [10-16] or (liquid) metallic [1,2,5,17-22] phases.

Accurate theoretical predictions of the equilibrium phase boundaries and other properties of high-pressure hydrogen require an appropriate treatment of quantum nuclear and many-electron correlation effects [23-28], which can be 
achieved only by using state-of-the-art $a b$ initio methods. Hitherto, most $a b$ initio studies of solid hydrogen have been based either on density functional theory (DFT) $[15,18,29,30]$ or quantum Monte Carlo calculations [24-26,31-34]. DFT employing approximate exchange and correlation (XC) energy functionals can be applied to compute infrared and Raman spectra as well as equilibrium phase boundaries, facilitating a direct comparison between theory and experiment [10-12,16,35-38]. However, different parametrizations of the XC functional in DFT yield inconsistent predictions [26,32,39]. Diffusion Monte Carlo (DMC) produces more reliable pressure temperature phase diagrams [24-26,33,34]. Furthermore, DMC can also be used to compute quasiparticle gaps, including nuclear quantum effects [40]. Recently, we showed that coupled cluster singles and doubles (CCSD) theory predicts static lattice enthalpies of solid hydrogen phases with high accuracy and computational efficiency [41]. CCSD results for the most stable model phases, including phases II and III, are in good agreement with those obtained using diffusion Monte Carlo. However, these studies are based on structures optimized using approximate XC functionals, causing uncontrollable errors when comparing computed transition pressures or band gaps to experiment. Here, we employ accurate many-electron theories to predict the atomic structure of crystalline molecular hydrogen phases and related properties, enabling a more rigorous study of band gaps and vibrational frequencies.

\section{METHODS}

We optimize the atomic structure of model phase III using nuclear gradients calculated on the level of second-order Møller-Plesset (MP2) perturbation theory and a plane wave basis set; see the Supplemental Material for implementation details [42]. We note there are some earlier implementations of MP2 forces in periodic solids using Gaussian basis set [43-45]. All periodic calculations have been performed using the Vienna Ab initio Simulation Package (VASP) [46] in the framework of the projector augmented wave method $[47,48]$, interfaced to our coupled cluster code [49] that employs an automated tensor contraction framework (CTF) [50]. We use Hartree-Fock orbitals in all post-Hartree-Fock methods [51]. Computational details are discussed in Ref. [42]. Although MP2 theory can be considered a low-order approximation to CCSD theory, it predicts lattice constants for a wide range of solids with higher accuracy than the DFT Perdew-BurkeErnzerhof (PBE) functional when compared to experiment [52]. Due to the many-electron nature of the employed ansatz, CCSD theory is exact for two-electron systems. The coupling between electron pairs is, however, approximated by truncating the many-body perturbation expansion in a computationally efficient manner and performing a resummation to infinite order of only certain contributions.

Phase III is modeled by $C 2 / c$ - 24 crystals [15] initially predicted by $a b$ initio simulations and random structure searches $[15,24]$. The structure is labeled by its symmetry followed by the number of atoms in the primitive cell. $C 2 / c-24$ consists of layered hydrogen molecules whose bonds lie within the plane of the layer, forming a distorted hexagonal shape. We note that previous DMC studies employed structures that have been optimized using a range of approximate density functionals, indicating that an appropriate choice is crucial [26]. In this work we employ supercells containing up to 96 atoms for the relaxation of the atomic positions. The convergence with respect to computational parameters such as the number of virtual orbitals, $k$ meshes for the Hartree-Fock energy contribution, and energy cutoffs for the employed plane wave basis set have been checked carefully and are summarized in the Supplemental Material [42].

Beyond the static lattice model, the $T$-dependent band gap renormalization of the single-particle excitation energy due to electron-phonon interactions (EPIs) was also studied, using a dynamical extension of the static EPI theory originally proposed by Heine, Allen, and Cardona (HAC) [53,54]. The quasiparticle approximation was used to correct the DFT-PBE eigenvalues based on the EPI self-energies. These calculations are performed using QUANTUM ESPRESSO [55] and YAMBO $[56,57]$. More details can be found in the Supplemental Material [42] and the reference therein [58]. The excitonic effects were obtained by solving the Bethe-Salpeter equation, as implemented in VASP. The EPIs and the excitonic effects are calculated using DFT-PBE optimized primitive cell structure.

\section{RESULTS}

\section{A. Structural optimization employing MP2 forces}

We fully relax the internal degrees of freedom of DFTPBE structures by minimizing the atomic forces computed on the level of MP2 theory while keeping the lattice vectors fixed and maintaining the space group symmetry. The MP2 structures are published in the Supplemental Material alongside additional results, demonstrating that further effects resulting from the relaxation of the lattice vectors can be disregarded [42]. For the purpose of the following discussion we will focus on the shortest hydrogen bond length in these structures, which represents the most striking difference between MP2 and DFT-PBE results. At a pressure of $250 \mathrm{GPa}$, the shortest hydrogen molecule bond length in the DFT-PBE structures for phase III is $0.75 \AA$, whereas MP2 theory predicts $0.72 \AA$. Similar findings apply to the structures at other pressures. In passing we note that the shortest hydrogen molecule bond length obtained using the van der Waals densityfunctional (vdW-DF) functional [59] is $0.72 \AA$, which is fortuitously close to our MP2 findings and agrees with findings reported in Ref. [26]. However, it is important to assess the reliability of these optimized structures further by comparing to CCSD results. Figure 1 illustrates that the total MP2 energy per atom of phase III at a volume of $1.57 \AA^{3} /$ atom (corresponding to a DFT-PBE pressure of $250 \mathrm{GPa}$ ) is lowered by about $5 \mathrm{meV} /$ atom during the structural relaxation. The initial 11 steps of the relaxation were carried out using only a 72-atom supercell, whereas all further optimization steps were performed using a 96-atom supercell, indicating that finite-size effects become negligible. The shortest bond length is changed by only about $0.01 \AA$ between the 11 th step and the final step. After 14 steps the remaining forces on the atoms are smaller than $0.05 \mathrm{eV} / \AA$. Figure 1 also depicts that the CCSD energy is decreased in total by $11 \mathrm{meV} /$ atom during the full MP2 relaxation trajectory, which is similar to the change in 


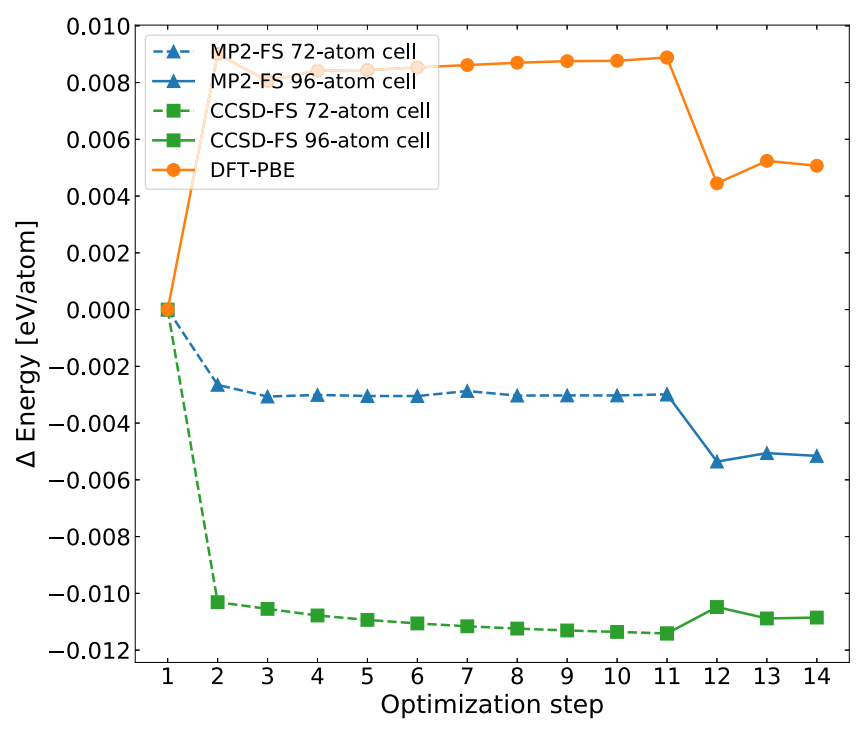

FIG. 1. The energy changes during the structural relaxation as a function of the optimization steps. This example at the DFT pressure of $250 \mathrm{GPa}$ shows that the MP2 and CCSD total energies per atom are lowered in a similar fashion and provides evidence that the optimized MP2 structures are close to the CCSD structures. The MP2 and CCSD energies are corrected by finite-size corrections [60] and are labeled by MP2-FS and CCSD-FS, respectively,

MP2 theory. The latter observation is important because it demonstrates that MP2 and CCSD equilibrium structures are expected to deviate only slightly. This justifies the main assumption of the present work which states that MP2 structures for phase III are very accurate. To further substantiate this claim, we note that MP2 theory predicts lattice constants for a wide range of solids with significantly higher accuracy than DFT-PBE when compared to experiment [52].

\section{B. DFT-PBE band structures}

As a first demonstration of the far-reaching consequences of the structural changes, we discuss their impact on the quasiparticle band gap of model phase III $(C 2 / c$-24). Figure 2 depicts the electronic band structure for phase III at a pressure of $250 \mathrm{GPa}$ employing the atomic structures optimized using DFT-PBE and MP2 theory. The Kohn-Sham band structures are computed using the PBE functional, exhibiting an indirect band gap with the valence band maximum at $X$ and the

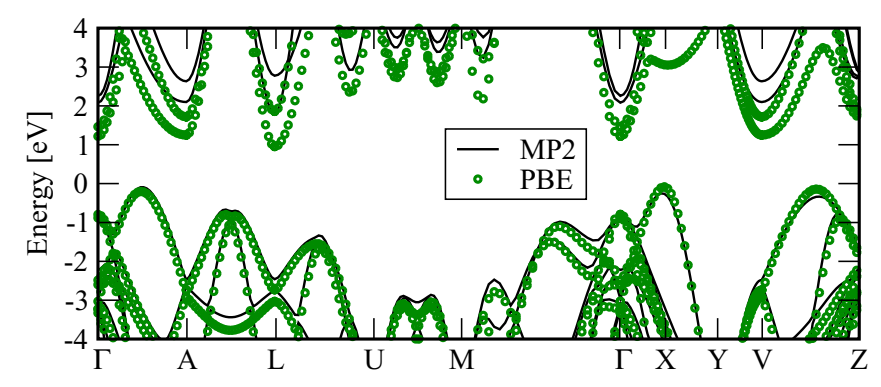

FIG. 2. Electronic band structure of model phase III $(C 2 / c-24)$ obtained using DFT-PBE. Black (green) lines correspond to MP2 (DFT-PBE) equilibrium geometries at a pressure of $250 \mathrm{GPa}$.

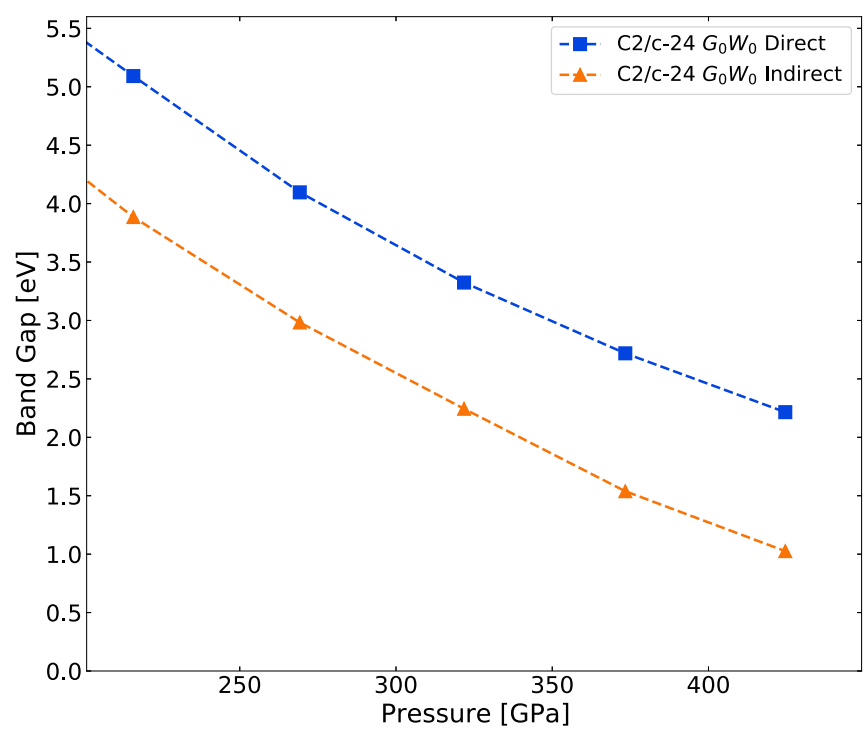

FIG. 3. Pressure dependence of the direct and indirect $G_{0} W_{0}$ band gaps of the ideal $C 2 / c$ - 24 crystal optimized by MP2 forces.

conduction band minimum at $L$. The direct gap is located at $\Gamma$. The direct and indirect PBE band gaps for the MP2 structure are 2.97 and $1.9 \mathrm{eV}$, respectively. However, due to the reduced hydrogen bond length, the direct and indirect band gaps are about $1 \mathrm{eV}$ larger in the MP2 structure than in the DFT-PBE structure.

\section{C. $G_{0} W_{0}$ band gaps of the static crystal}

We note that this increase in the band gap persists for the more accurate quasiparticle band gaps computed on the level of the $G_{0} W_{0}$ approximation (see Fig. 3). We stress that due to the strong dependence of the electronic gap on the pressure, an underestimation of the band gap by $1 \mathrm{eV}$ results in a decrease in the predicted metallization pressure by more than $50 \mathrm{GPa}$. We note that the previously employed vdW-DF structures in Refs. [26,40] yield band gaps that agree with our findings obtained using the MP2 structures to within about $0.1 \mathrm{eV}$. The direct and indirect PBE band gaps computed using the vdW-DF structures are 2.88 and $1.74 \mathrm{eV}$, respectively.

\section{Renormalized $G_{0} W_{0}$ band gaps by electron-phonon interactions}

We now turn to the comparison between computed $G_{0} W_{0}$ band gaps and experimental findings. As shown in Ref. [40], the inclusion of zero-point vibrational effects in the quasiparticle gaps is crucial. At $0 \mathrm{~K}$, this effect is termed zero-point renormalization (ZPR). At finite $T \mathrm{~s}, T$-dependent band gap renormalization also exists, originating from the Fan and Debye-Waller terms as described in the dynamical HAC theory. More details can be found in Refs. [57,61]. Unfortunately, seamless inclusion of the electron-phonon coupling contributions to the band gap on the level of MP2 theory would be computationally too expensive at the moment. Therefore, we estimate these renormalizations using DFT-PBE phonons and include them in the $G_{0} W_{0}$ quasiparticle band gaps [42]. Our calculations yield a ZPR of the direct and indirect 


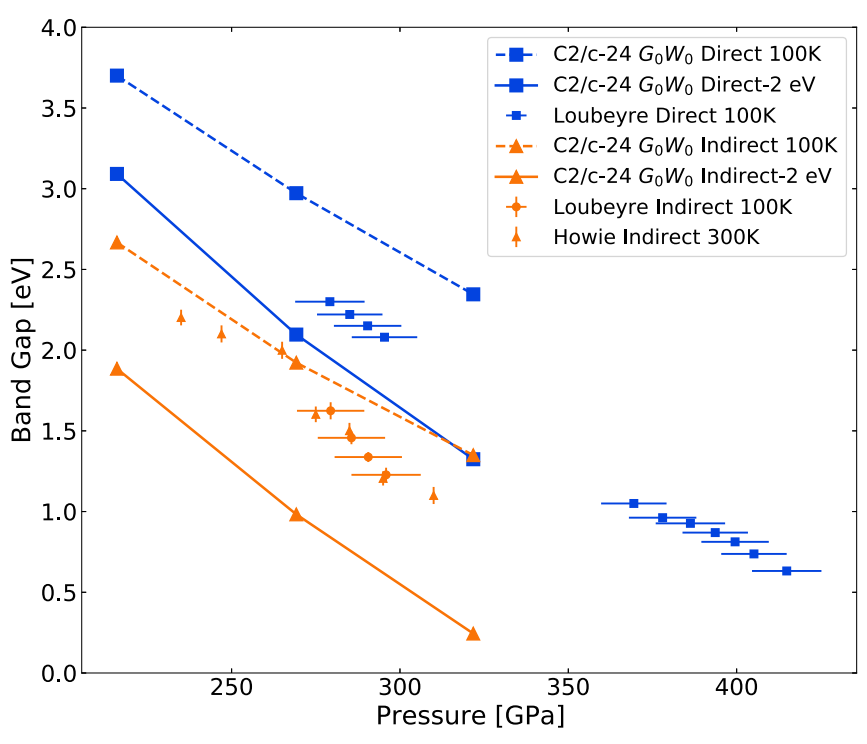

FIG. 4. Pressure dependence of the direct and indirect $G_{0} W_{0}$ band gaps including EPI contributions from this work (dashed lines) and $\approx-2 \mathrm{eV}$ EPI contributions from Ref. [40] (solid lines). The direct $G_{0} W_{0}$ band gaps include $\approx-0.12 \mathrm{eV}$ exciton binding energy. The experimental estimates have been taken from Refs. $[4,7,16,40]$.

gaps of about $-1 \mathrm{eV}$, which is, by coincidence, a magnitude similar to the band gap increase caused by structural relaxation but significantly smaller than the $-2 \mathrm{eV}$ ZPR reported previously [40].

The computed $G_{0} W_{0}$ gaps with EPIs are depicted in Fig. 4 for a range of pressures alongside experimental findings $[4,7,16,40]$. We note that the direct $G_{0} W_{0}$ gaps include $\approx-0.12 \mathrm{eV}$ exciton binding energy [42] in order to enable a direct comparison to the optical measurements from Refs. [4,7]. Furthermore, we plot the $G_{0} W_{0}$ gaps with respect to the CCSD pressures computed from the enthalpy versus volume curves, enabling an accurate and direct comparison to experimental findings. Compared to experiments, the direct and indirect quasiparticle band gaps are overestimated when our EPI values are used. Replacing our EPI contribution with the estimate by Gorelov et al. $(\approx-2 \mathrm{eV})$ yields underestimated band gaps compared to experiment. From the relatively large difference between the EPI contributions computed in this work and Ref. [40], we conclude that this contribution is the remaining leading-order error in our study. However, the experimental metallization pressure of about $450 \mathrm{GPa}$ lies within our theoretical uncertainties. In a recent work by Monacelli et al. [62], quantum nuclear effects were included, which showed a reduction of the band gaps by approximately $3 \mathrm{eV}$. Their metallization pressure agrees well with experiment. Their structures, however, are optimized on the level of DFT using the Becke-Lee-Yang-Parr exchangecorrelation functional. Another study on the band gap that achieved very good agreement with experiment was carried out by Dogan et al. [63] using DFT-PBE optimized and static crystal structures. From the above analysis, we expect that this good agreement is probably the result of fortuitous error cancellation due to the two compensating effects: (i) the underestimation of the band gap by using the DFT-PBE struc- tures and (ii) not taking into account EPIs which reduce the band gap.

\section{E. $\boldsymbol{T}$-dependent direct and indirect band gap renormalizations}

The difference in the $T$-dependent indirect band gap renormalizations in Ref. [40] between 200 and $300 \mathrm{~K}$ is about $0.2 \mathrm{eV}$, which is an order of magnitude larger than our estimate of $0.02 \mathrm{eV}$ (see Fig. 5). The difference in the experimental indirect band gaps between $100 \mathrm{~K}$ [4] and $300 \mathrm{~K}$ [16] is about $0.02 \mathrm{eV}$, which agrees much better with our result. These discrepancies signal that further careful examinations of the employed structures in different studies and the treatment of different contributions are needed.

\section{F. Pressure dependence of $\mathrm{H}_{2}$ vibron frequencies}

For a deeper understanding of the comparison with experiments, we also assess the reliability of the experimental pressure calibration. This is done by analyzing the dependence of the $\mathrm{H}_{2}$ vibron peak frequency as a function of the pressure. As pointed out in Refs. [7,9,64] and depicted in Fig. 6, the currently available experimental estimates for the $\mathrm{H}_{2}$ vibron peak frequency vary significantly at high pressures, questioning the reliability of experimentally determined pressures. Possible reasons for the experimental uncertainties are summarized in Ref. [9]. However, theoretical estimates of the vibron peak frequency with respect to pressure also vary significantly with respect to the employed XC parametrization on the level of DFT [7,26]. We have estimated the vibrational frequency for the MP2 structures by computing the MP2 and CCSD energies as a function of $\mathrm{H}_{2}$ bond lengths around the equilibrium. Molecular orientations, locations of the centers of mass, and volumes are fixed while changing the bond lengths in accordance with Ref. [26]. The change in the harmonic frequency with respect to the pressure can be used as a reliable calibration for pressures depicted in Fig. 6. We find that both the MP2 and CCSD frequencies have a slope similar to the $\mathrm{H}_{2}$ vibron frequency peak measured by Loubeyre et al. in Refs. [7,65]. From this we conclude that the experimental band gaps depicted in Fig. 4 correspond to pressures that are in good agreement with our most accurate estimates. In passing we note that despite the good agreement of vdW-DF structures with our MP2 structures, vdW-DF vibrational frequencies are in better agreement with experimental results of Refs. [35,64]. However, we argue that this agreement is most likely fortuitous because both MP2 and the more accurate CCSD vibrational frequencies exhibit a very similar and steeper slope with respect to pressure. From the above findings, we conclude that the vibrational frequencies of high-pressure hydrogen phases are very sensitive to the structural parameters and the corresponding electronic structure method. This has potential implications for estimates of the zero-point motion energy contribution to the lattice enthalpies of accurate $a b$ initio calculations of transition pressures [24]. Having established the good agreement between our pressure estimates and those reported in Ref. [7], we can also comment on the observed evidence of a phase transition at $425 \mathrm{GPa}$. As predicted by both DMC and CCSD calculations $[24,26,41]$ at low temperature, phase III $(C 2 / c-24)$ 

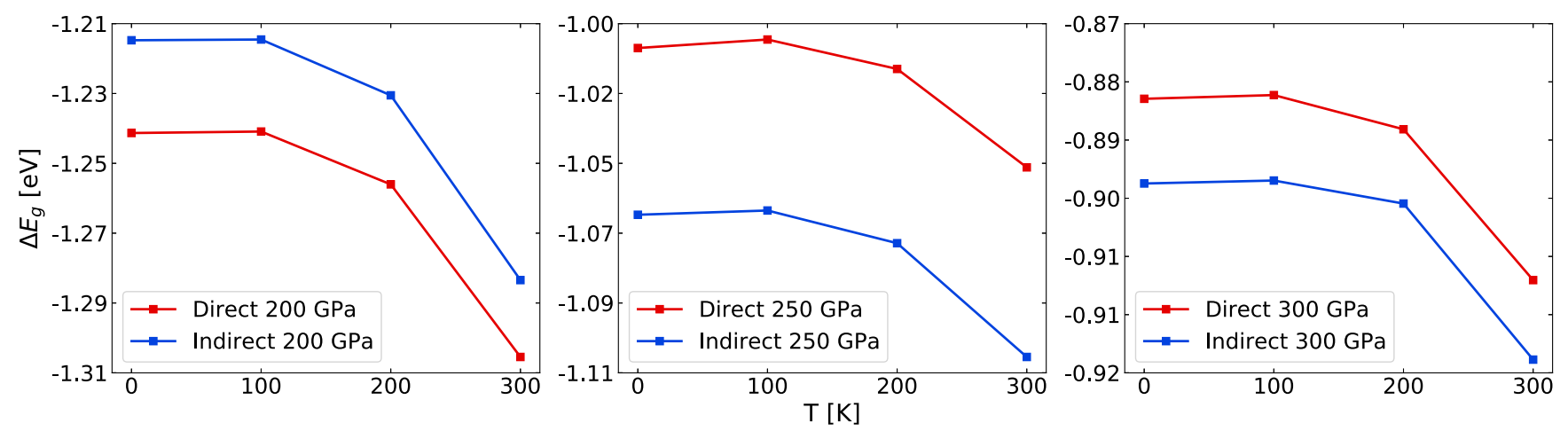

FIG. 5. $T$-dependent renormalizations due to EPIs on the direct and indirect band gaps of model phase III $(C 2 / c$-24) obtained using DFT-PBE at 200, 250, and 300GPa, calculated using dynamical HAC theory. 100 random $q$ points, 300 electronic bands, and a $4 \times 4 \times 4 k$ grid are used.

transforms into Cmca-12 at this pressure. However, these calculations were performed using DFT optimized structures. We have investigated the lowering of static lattice enthalpies resulting from MP2 lattice relaxations for both structures at a selected volume corresponding to a DFT pressure of $450 \mathrm{GPa}$, finding that changes to the previously calculated transition pressures are negligible. This is surprising given the relatively large changes in the $\mathrm{H}_{2}$ bond lengths.

\section{CONCLUSIONS}

Our work demonstrated the strengths and weaknesses of widely used approximate DFT methods for simulating highpressure phases of hydrogen by comparing them to more

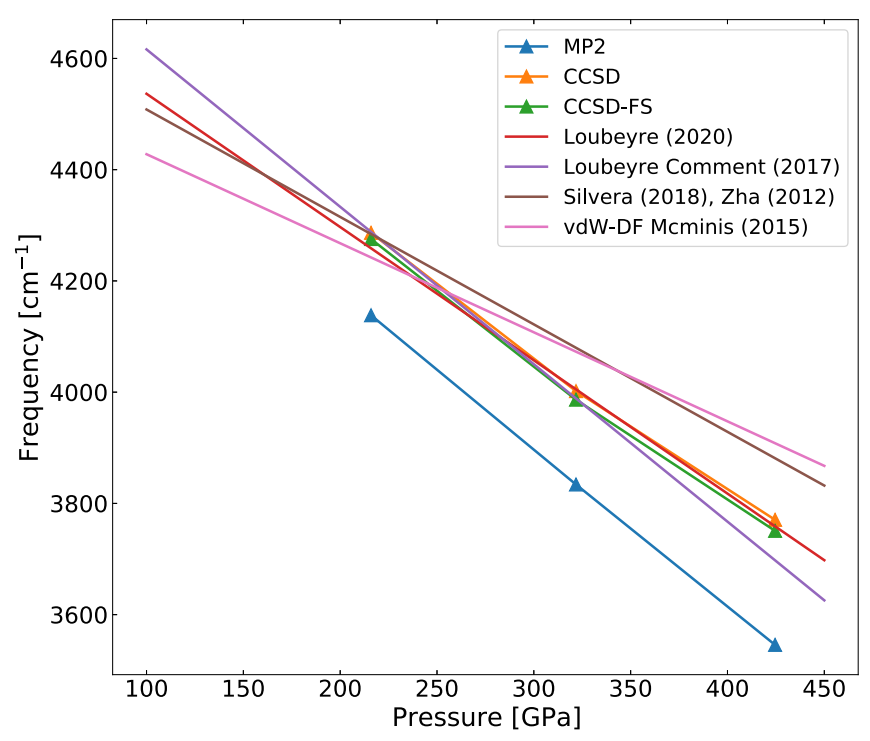

FIG. 6. Experimentally measured and theoretically calculated $\mathrm{H}_{2}$ vibron peak frequencies as a function of pressure. The approximate vdW-DF (taken from the Supplemental Material of Ref. [26]), MP2, and CCSD harmonic frequencies are shown by the pink, blue, and green solid lines, respectively. The brown solid line shows the experimentally measured relation between the $\mathrm{H}_{2}$ vibron frequencies and pressures from Silvera and Dias [64] and Zha [35]. Another two experimental data lines are from Loubeyre et al. from 2020 [7] (red) and from 2017 [65] (purple). accurate results obtained using many-electron methods including coupled cluster theory. Although approximate density functional theory is a computationally efficient tool for performing random structure searches [15], further structural optimization is required to achieve good agreement of band gaps and vibrational frequencies with experimental findings in solid hydrogen. Here, we demonstrated that periodic many-electron perturbation theory calculations using plane wave basis sets have become increasingly efficient in previous years $[41,60]$, making such optimizations feasible for systems with an increasing number of atoms. Our findings show that compared to MP2 theory, DFT-PBE structures exhibit too large hydrogen bond lengths, causing too small band gaps. Although vdW-DF calculations predict structures that are closer to MP2 theory, vibrational frequencies that agree with experiment for a wide range of pressures can be obtained only on the level of CCSD. Furthermore, we have demonstrated that the remaining leading-order error of $a b$ initio band gaps in solid hydrogen crystals is likely to originate from approximations used to estimate the EPI contributions. Nevertheless, it is worth pointing out that $T$ dependent fundamental band gap renormalization based on DFT-PBE structure is in better agreement with the experimental data. Combining accurate benchmark results with hybrid or nonlocal XC functionals using adjustable parameters could be useful for materials modeling in this case. Alternatively, machine learning from MP2 forces or even more accurate $a b$ initio data could be used to produce accurate potential energy surfaces and corresponding vibrational entropy contributions. Future work will focus on a seamless integration of electron-phonon interaction on the level of many-electron theories to further improve the accuracy of such $a b$ initio simulations.

\section{ACKNOWLEDGMENTS}

K.L. thanks D. Kats for useful discussions in the early stage of the project and the Max Planck Institute for Solid State Research for the Ph.D. funding. T.S. wants to thank X.-W. Zhang and Q.-J. Ye for fruitful discussions. Support and funding from the European Research Council (ERC) under the European Union's Horizon 2020 research and innovation program (Grant Agreement No. 715594); the 
National Science Foundation of China under Grants No. 11934003, No. 11774003, and No. 11634001; Beijing Natural Science Foundation under Grant No. Z200004; the Strategic Priority Research Program of the Chinese Academy of Sciences under Grant No. XDB33010400; and the National Basic Research Programs of China under Grant No.
2016YFA0300901 are gratefully acknowledged. The computational results presented were achieved using the Vienna Scientific Cluster (VSC), the cluster of the Electronic Structure Theory Department at Max Planck Institute for Solid State Research, and the High-performance Computing Platform of Peking University, China.
[1] E. Wigner and H. B. Huntington, J. Chem. Phys. 3, 764 (1935).

[2] R. J. Hemley and H.-K. Mao, Science 244, 1462 (1989).

[3] C. Narayana, H. Luo, J. Orloff, and A. L. Ruoff, Nature (London) 393, 46 (1998).

[4] P. Loubeyre, F. Occelli, and R. LeToullec, Nature (London) 416, 613 (2002).

[5] R. P. Dias and I. F. Silvera, Science 357, eaao5843 (2017).

[6] R. P. Dias and I. F. Silvera, Science 355, 715 (2017)

[7] P. Loubeyre, F. Occelli, and P. Dumas, Nature (London) 577, 631 (2020).

[8] H. Y. Geng, Matter Rad. Extremes 2, 275 (2017).

[9] I. F. Silvera and R. Dias, arXiv:1907.03198.

[10] R. J. Hemley and H. K. Mao, Phys. Rev. Lett. 61, 857 (1988).

[11] H. E. Lorenzana, I. F. Silvera, and K. A. Goettel, Phys. Rev. Lett. 63, 2080 (1989).

[12] H. K. Mao and R. J. Hemley, Rev. Mod. Phys. 66, 671 (1994).

[13] V. Natoli, R. M. Martin, and D. Ceperley, Phys. Rev. Lett. 74, 1601 (1995).

[14] I. Goncharenko and P. Loubeyre, Nature (London) 435, 1206 (2005).

[15] C. J. Pickard and R. J. Needs, Nat. Phys. 3, 473 (2007).

[16] R. T. Howie, C. L. Guillaume, T. Scheler, A. F. Goncharov, and E. Gregoryanz, Phys. Rev. Lett. 108, 125501 (2012).

[17] S. T. Weir, A. C. Mitchell, and W. J. Nellis, Phys. Rev. Lett. 76, 1860 (1996).

[18] K. A. Johnson and N. W. Ashcroft, Nature (London) 403, 632 (2000).

[19] M. I. Eremets and I. A. Troyan, Nat. Mater. 10, 927 (2011).

[20] J. Chen, X.-Z. Li, Q. Zhang, M. I. J. Probert, C. J. Pickard, R. J. Needs, A. Michaelides, and E. Wang, Nat. Commun. 4, 2064 (2013).

[21] M. D. Knudson, M. P. Desjarlais, A. Becker, R. W. Lemke, K. R. Cochrane, M. E. Savage, D. E. Bliss, T. R. Mattsson, and R. Redmer, Science 348, 1455 (2015).

[22] M. Zaghoo, A. Salamat, and I. F. Silvera, Phys. Rev. B 93, 155128 (2016).

[23] X.-Z. Li, B. Walker, M. I. J. Probert, C. J. Pickard, R. J. Needs, and A. Michaelides, J. Phys.: Condens. Matter 25, 085402 (2013).

[24] N. D. Drummond, B. Monserrat, J. H. Lloyd-Williams, P. L. Ríos, C. J. Pickard, and R. J. Needs, Nat. Commun. 6, 7794 (2015).

[25] S. Azadi, W. M. C. Foulkes, and T. D. Kühne, New J. Phys. 15, 113005 (2013).

[26] J. McMinis, R. C. Clay, D. Lee, and M. A. Morales, Phys. Rev. Lett. 114, 105305 (2015).

[27] M. A. Morales, J. M. McMahon, C. Pierleoni, and D. M. Ceperley, Phys. Rev. B 87, 184107 (2013).

[28] M. A. Morales, J. M. McMahon, C. Pierleoni, and D. M. Ceperley, Phys. Rev. Lett. 110, 065702 (2013).
[29] H. Chacham and S. G. Louie, Phys. Rev. Lett. 66, 64 (1991).

[30] J. Kohanoff, S. Scandolo, G. L. Chiarotti, and E. Tosatti, Phys. Rev. Lett. 78, 2783 (1997).

[31] D. M. Ceperley and B. J. Alder, Phys. Rev. B 36, 2092 (1987).

[32] R. C. Clay, III, J. Mcminis, J. M. McMahon, C. Pierleoni, D. M. Ceperley, and M. A. Morales, Phys. Rev. B 89, 184106 (2014).

[33] S. Azadi, B. Monserrat, W. M. C. Foulkes, and R. J. Needs, Phys. Rev. Lett. 112, 165501 (2014).

[34] S. Azadi, N. D. Drummond, and W. M. C. Foulkes, Phys. Rev. B 95, 035142 (2017).

[35] C.-S. Zha, Z. Liu, and R. J. Hemley, Phys. Rev. Lett. 108, 146402 (2012).

[36] M. Hanfland, R. J. Hemley, and H.-K. Mao, Phys. Rev. Lett. 70, 3760 (1993).

[37] B. Monserrat, R. J. Needs, E. Gregoryanz, and C. J. Pickard, Phys. Rev. B 94, 134101 (2016).

[38] X.-W. Zhang, E.-G. Wang, and X.-Z. Li, Phys. Rev. B 98, 134110 (2018).

[39] S. Azadi and G. J. Ackland, Phys. Chem. Chem. Phys. 19, 21829 (2017).

[40] V. Gorelov, M. Holzmann, D. M. Ceperley, and C. Pierleoni, Phys. Rev. Lett. 124, 116401 (2020).

[41] K. Liao, X.-Z. Li, A. Alavi, and A. Grüneis, npj Comput. Mater. 5, 110 (2019).

[42] See Supplemental Material at http://link.aps.org/supplemental/ 10.1103/PhysRevB.103.054111, which includes the optimized crystal structures, details of the implementation and additional calculation details.

[43] M. Del Ben, J. Hutter, and J. VandeVondele, J. Chem. Phys. 143, 102803 (2015).

[44] F. Weigend and M. Häser, Theor. Chem. Acc. 97, 331 (1997).

[45] V. V. Rybkin and J. VandeVondele, J. Chem. Theory Comput. 12, 2214 (2016).

[46] G. Kresse and J. Furthmüller, Phys. Rev. B 54, 11169 (1996).

[47] P. E. Blöchl, Phys. Rev. B 50, 17953 (1994).

[48] G. Kresse and D. Joubert, Phys. Rev. B 59, 1758 (1999).

[49] F. Hummel, T. Tsatsoulis, and A. Grüneis, J. Chem. Phys. 146, 124105 (2017).

[50] E. Solomonik, D. Matthews, J. R. Hammond, J. F. Stanton, and J. Demmel, J. Parallel Distributed Comput. 74, 3176 (2014).

[51] A. Grüneis, G. H. Booth, M. Marsman, J. Spencer, A. Alavi, and G. Kresse, J. Chem. Theory Comput. 7, 2780 (2011).

[52] A. Grüneis, M. Marsman, and G. Kresse, J. Chem. Phys. 133, 074107 (2010).

[53] P. B. Allen and V. Heine, J. Phys. C 9, 2305 (1976).

[54] P. B. Allen and M. Cardona, Phys. Rev. B 27, 4760 (1983).

[55] P. Giannozzi, S. Baroni, N. Bonini, M. Calandra, R. Car, C. Cavazzoni, D. Ceresoli, G. L. Chiarotti, M. Cococcioni, I. Dabo, and A. Dal Corso, J. Phys. Condens. Matter 21, 395502 (2009). 
[56] A. Marini, C. Hogan, M. Grüning, and D. Varsano, Comput. Phys. Commun. 180, 1392 (2009).

[57] D. Sangalli, A. Ferretti, H. Miranda, C. Attaccalite, I. Marri, E. Cannuccia, P. Melo, M. Marsili, F. Paleari, A. Marrazzo, G. Prandini, P. Bonfa, M. O. Atambo, F. Affinito, M. Palummo, A. Molina-Sanchez, C. Hogan, M. Gruning, D. Varsano, and A. Marini, J. Phys.: Condens. Matter 31, 325902 (2019).

[58] D. R. Hamann, Phys. Rev. B 88, 085117 (2013).

[59] K. Lee, E. D. Murray, L. Kong, B. I. Lundqvist, and D. C. Langreth, Phys. Rev. B 82, 081101(R) (2010).

[60] T. Gruber, K. Liao, T. Tsatsoulis, F. Hummel, and A. Grüneis, Phys. Rev. X 8, 021043 (2018).
[61] T. Shen, X.-W. Zhang, H. Shang, M.-Y. Zhang, X. Wang, E.-G. Wang, H. Jiang, and X.-Z. Li, Phys. Rev. B 102, 045117 (2020).

[62] L. Monacelli, I. Errea, M. Calandra, and F. Mauri, Nat. Phys. 17, 63 (2021).

[63] M. Dogan, S. Oh, and M. L. Cohen, J. Phys.: Condens. Matter 33, 03LT01 (2020).

[64] I. F. Silvera and R. Dias, J. Phys.: Condens. Matter 30, 254003 (2018).

[65] P. Loubeyre, F. Occelli, and P. Dumas, Science 357, eaan2286 (2017).

Correction: The previously published Figure 6 contained an incorrect ordinate label and has been replaced. 